\title{
Spectral Stochastic Finite Element Method for Electromagnetic Problems with Random Geometry
}

\author{
Antti Lehikoinen (Doctoral student, Aalto University)
}

\begin{abstract}
In electromagnetic problems, the problem geometry may not always be exactly known. One example of such a case is a rotating machine with random-wound windings. While spectral stochastic finite element methods have been used to solve statistical electromagnetic problems such as this, their use has been mainly limited to problems with uncertainties in material parameters only. This paper presents a simple method to solve both static and time-harmonic magnetic field problems with source currents in random positions. By using an indicator function, the geometric uncertainties are effectively reduced to material uncertainties, and the problem can be solved using the established spectral stochastic procedures. The proposed method is used to solve a demonstrative single-conductor problem, and the results are compared to the Monte Carlo method. Based on these simulations, the method appears to yield accurate mean values and variances both for the vector potential and current, converging close to the results obtained by time-consuming Monte Carlo analysis. However, further study may be needed to use the method for more complicated multi-conductor problems and to reduce the sensitivity of the method on the mesh used.
\end{abstract}

Keywords - Eddy currents; Finite element analysis; Stochastic systems; Random variables.

\section{INTRODUCTION}

To design efficient electrical machines, methods are needed to accurately solve two-dimensional electromagnetic problems. Finite element methods do fill this requirement, but have mostly been used to solve purely deterministic problems with exactly known parameters. However, a designer may often encounter cases where one or more problem parameter is random and not exactly known. For instance, the permeability of iron or other material parameters may be changed due to manufacture [1]. The exact problem geometry can also be uncertain. For example, in random-wound stator windings, the exact positions of the conductors are unknown, leading to circulating current losses that are very difficult to predict [2]. Indeed, most authors have chosen to assume a particular deterministic conductor transposition before loss calculation [3]- [6].

Different Monte Carlo (MC) methods can be used to solve stochastic problems like these. In these methods, a deterministic problem is solved repeatedly with randomly assigned parameters. The stochastic behavior of the solution is then approximated from the obtained deterministic solutions. While this method is easy to understand and implement, it suffers from very long computation times due to the large number of simulations needed. [7]

One solution to long computation times is the spectral stochastic finite element method (ssFEM). With ssFEM, it is sufficient to solve a larger deterministic problem once. Indeed, the method has been used to solve different stochastic electrostatic and electromagnetic problems, although mainly with uncertain material parameters rather than an uncertain geometry. [8] - [10]

Some attempts to use the spectral stochastic FEM also with geometrically uncertain problems have been made. A method based on a random domain mapping was presented in [11] [12]. While the method appears suitable for a wide range of problems, it is relatively complicated. Moreover, a specific mapping has to be found for each problem. Finally, its suitability for problems with large or numerous variations in geometry has not yet been demonstrated.

This paper presents a simpler method to solve electromagnetic problems with current sources on random positions. The uncertainties in the geometry are expressed with an integer-valued indicator function, effectively reducing the problem to one with only material uncertainties. A solution to the electromagnetic problem is then obtained with the typical spectral stochastic solution procedure. The proposed method is then used to solve a simplified problem of a conductor in a slot at a random location. Problems both with and without eddy currents are solved.

\section{THEORY}

The proposed spectral stochastic finite element method is derived in this section. First, the necessary theory of the polynomial chaos expansion is briefly presented, based on [7] and [13]. Derivation of the actual solution method follows thereafter.

\section{A. Polynomial Chaos Expansion}

The polynomial chaos expansion is a method to present a random variable as a sum of functionals of other random variables. It is similar for instance to the Fourier transformation, performed in probability space instead of frequency domain. In a one-dimensional case, the method can be presented as follows.

Let $Y$ be a random variable. The polynomial chaos expansion of $Y$ is then

$$
Y=Y(\theta) \approx \sum_{j=0}^{P} y_{j} \Psi_{j}(X(\theta)) .
$$

Here, $X$ is a random variable with a known probability density function $\mathrm{f}(x)$ in the interval $[a, b] . P$ is the order of the expansion, and the $y_{j}$ terms are the expansion coefficients. 
The $\Psi_{j}$ terms are $j^{\text {th }}$ order polynomials of $X$, typically called polynomial chaoses. They are normally chosen to be orthogonal with respect to the expectation

$$
\begin{array}{r}
\mathrm{E}\left[\Psi_{i}(X(\theta)) \Psi_{j}(X(\theta))\right] \\
=\int_{a}^{b} f(x) \Psi_{i}(x) \Psi_{j}(x) \mathrm{d} x \\
=0 \forall i \neq j .
\end{array}
$$

For instance, if $X$ follows the normal distribution, Hermite polynomials satisfy this orthogonality requirement.

The coefficients $y_{j}$ can then be calculated as follows. Let $\mathrm{G}$ be a mapping from $X$ to $Y$. In other words, G must satisfy the equality

$$
Y(\theta)=\mathrm{G}(X(\theta)) \quad \forall \theta
$$

With this definition, the coefficients can then be calculated with the Galerkin projection [14]

$$
y_{j}=\frac{\mathrm{E}\left[\mathrm{G}(X(\theta)) \Psi_{j}(X(\theta))\right]}{\mathrm{E}\left[\Psi_{j}(X(\theta)) \Psi_{j}(X(\theta))\right]} .
$$

In a multi-dimensional case, the probability space is spanned by several uncorrelated random variables $X_{1}, X_{2}, \ldots$, $X_{M}$. In this case, the polynomial chaoses can be obtained as products of one-dimensional chaoses of one variable, in other words as

$$
\begin{array}{r}
\Psi_{i}\left(X_{1}, X_{2}, \ldots X_{M}\right) \\
=\Psi_{\alpha_{1}^{i}}\left(X_{1}\right) \Psi_{\alpha_{2}^{i}}\left(X_{2}\right) \ldots \Psi_{\alpha_{2}^{i}}\left(X_{M}\right) .
\end{array}
$$

The orders $\alpha$ must fulfil the criterion

$$
\sum_{k=1}^{M} \alpha_{k}^{i} \leq P \quad \forall i .
$$

Naturally, the orders have to be chosen so that a linearly independent set of chaoses is obtained. In other words, no repetition in the products can take place. The expectation (2) can be generalized to multiple dimensions in a similar fashion.

\section{B. Static Magnetic Field Problem in a Deterministic Case}

Suppose that a solution to a linear static two-dimensional magnetic field problem on a domain $\Omega$ is sought. $\Omega$ is assumed to lie in the xy-plane. By starting from the vector potential formulation of the Maxwell's equations

$$
\begin{aligned}
& \nabla \times \mathbf{H}(\mathbf{x})=J(\mathbf{x}) \mathbf{e}_{\mathrm{z}} \\
& \mathbf{H}(\mathbf{x})=v(\mathbf{x}) \mathbf{B}(\mathbf{x}) \\
& \mathbf{B}(\mathbf{x})=\nabla \times A(\mathbf{x}) \mathbf{e}_{\mathrm{z}},
\end{aligned}
$$

the following equation can be obtained

$$
\nabla \cdot(v(\mathbf{x}) \nabla A(\mathbf{x}))=-J(\mathbf{x}) .
$$

In the equations, $\mathbf{x}=\left[\begin{array}{ll}\mathrm{x} y & \mathrm{~T}\end{array}{ }^{\mathrm{T}}\right.$ is the position vector. $\mathbf{H}$ and $\mathbf{B}$ are the magnetic field strength and flux density vectors in the problem plane. $J$ is the magnitude of the source current density perpendicular to the problem domain. $A$ is the amplitude of the vector potential, and $v$ is the magnetic reluctivity.

For simplicity, $\Omega$ can be assumed to cover the entire problem region, so that no flux leaves the area. In this case, the weak form of the problem can be obtained by multiplying both sides of (8) with a test function $g$ and integrating by parts, which yields

$$
\begin{array}{r}
\int_{\Omega}(v(\mathbf{x}) \nabla A(\mathbf{x})) \cdot \nabla g(\mathbf{x}) \mathrm{d} \Omega \\
=\int_{\Omega} J(\mathbf{x}) g(\mathbf{x}) \mathrm{d} \Omega .
\end{array}
$$

In a more general case, a boundary term would also have to be considered. In this case, however, the vector potential on the boundary can be set to be zero, making the associated integral term disappear.

\section{Spectral Stochastic Finite Element Formulation with Geometric Uncertainty}

Equation (8) can be solved with the finite element method. In the deterministic case, the vector potential $A$ is approximated with a weighted sum of shape functions.

$$
A(\mathbf{x}) \approx \sum_{i=1}^{N} A_{i} \varphi_{i}(\mathbf{x}) .
$$

The constant coefficients $A_{i}$ are then solved with a suitable method. [14]

However, if there are uncertainties in the problem, each of the coefficients in (10) will be a random variable $A_{i}(\theta)$ rather than a constant. In the spectral stochastic finite element method, the coefficients $A_{i}$ are written as their respective polynomial chaos expansions

$$
A_{i}(\theta) \approx \sum_{j=0}^{P} A_{i j} \Psi_{j}(X(\theta)) .
$$

Here, $X$ can either be a single random variable, or a vector of uncorrelated random variables, depending on the problem to be solved. With this formulation, the remaining task is now to solve the coefficients $A_{i j}$. This subject will be shortly dealt with.

Before that, suppose that the source current density in (8) is due to a single conductor, the position of which is not exactly known. In other words, it is assumed that the current density $J$ gets a constant, nonzero value on a random subdomain $\Omega_{\theta}$, and is zero everywhere else. This situation can be expressed as

$$
J(\mathbf{x})=J_{0} \mathrm{D}_{\theta}(\mathbf{x}, X(\theta)) .
$$

Here, $D_{\theta}$ is an indicator function that gets a value of 1 if $\mathbf{x}$ belongs to the random domain $\Omega_{\theta}$, and 0 otherwise. For simplicity, it has been assumed that the subdomain $\Omega_{\theta}$, and by extension $\mathrm{D}_{\theta}$, can be written with the random variable $X$. For example, for a circular conductor with a radius of $r_{\mathrm{c}}$ and its center at $X=\left[X_{1} X_{2}\right]^{\mathrm{T}}, \mathrm{D}_{\theta}$ would be

$$
\mathrm{D}_{\theta}(\mathbf{x}, X(\theta))=\left\{\begin{array}{cc}
1, & |\mathbf{x}-X(\theta)| \leq r_{\mathrm{c}} \\
0, & \text { otherwise. }
\end{array}\right.
$$

With these formulations, the original problem can finally be solved. Dependencies on $\mathbf{x}$ and $\theta$ will be dropped for clarity. By choosing

$$
g=\Psi^{q} \varphi_{n}
$$


as the test function, and by substituting (11) and (12) back to (8), the following equality is obtained

$$
\begin{array}{r}
\int_{\Omega}\left(v \nabla \sum_{i=1}^{N} \sum_{j=0}^{P} A_{i j} \Psi_{j} \varphi_{i}\right) \cdot\left(\nabla \Psi_{q} \varphi_{n}\right) \mathrm{d} \Omega \\
=\int_{\Omega} J_{0} \mathrm{D}_{\theta} \Psi_{q} \varphi_{n} \mathrm{~d} \Omega .
\end{array}
$$

This is not yet a very useful form, since both sides of the equation are still random variables. By taking an expectation of the both sides, the following form is obtained after some simple reordering

$$
\begin{array}{r}
\sum_{i=1}^{N} \sum_{j=0}^{P} A_{i j} \mathrm{E}\left(\Psi_{j} \Psi_{q}\right) \int_{\Omega}\left(v \nabla \varphi_{i}\right) \cdot\left(\nabla \varphi_{n}\right) \mathrm{d} \Omega \\
=\int_{\Omega} J_{0} \mathrm{E}\left(\mathrm{D}_{\theta} \Psi_{q}\right) \varphi_{n} \mathrm{~d} \Omega .
\end{array}
$$

Obviously, this equality is purely deterministic, i.e. there is no randomness. Therefore, it can be solved with simple linear algebra.

The term on the right-hand side might be difficult to calculate analytically, since the $\mathrm{D}_{\theta}$ term is a function of both the position $\mathbf{x}$ and the random variable $X$. However, most numerical methods would approximate the integral as a finite sum

$$
\begin{array}{r}
\int_{\Omega} J_{0} \mathrm{E}\left(\mathrm{D}_{\theta} \Psi_{q}\right) \varphi_{n} \mathrm{~d} \Omega \\
\approx \sum_{k=1}^{K} J_{0} \mathrm{E}\left(\mathrm{D}_{\theta}\left(\mathbf{x}_{k}, X(\theta)\right) \Psi_{q}\right) \varphi_{n}\left(\mathbf{x}_{k}\right) .
\end{array}
$$

The points $\mathbf{x}_{\mathrm{k}}$ are the summation points specific to the integration method used. In this case, the expectation only has to be calculated for specific values of $\mathbf{x}$. This should pose no problems.

After these considerations, the problem can be easily formulated as a matrix equation. By fixing the values of $q$ and $n$ in (16), the following equality is obtained

$$
\begin{array}{r}
\mathrm{E}\left(\Psi_{q} \Psi_{q}\right) \sum_{i=1}^{N} A_{i q} \int_{\Omega}\left(v \nabla \varphi_{i}\right) \cdot\left(\nabla \varphi_{n}\right) \mathrm{d} \Omega \\
=\int_{\Omega} J_{0} \mathrm{E}\left(\mathrm{D}_{\theta} \Psi_{q}\right) \varphi_{n} \mathrm{~d} \Omega .
\end{array}
$$

The summation with respect to $j$ has been dropped since the expectation term will be zero for all $j \neq q$ due to the orthogonality of $\Psi_{\mathrm{j}}$. By letting $n$ in (18) run from 1 to $N$, the following matrix equation is obtained

$$
\mathbf{S}^{q} \mathbf{A}^{q}=\mathbf{F}^{q} \text {. }
$$

Here, $\mathbf{F}^{q}$ is the load vector with elements

$$
\left[\mathbf{F}^{q}\right]_{i}=\int_{\Omega} J_{0} \mathrm{E}\left(\mathrm{D}_{\theta} \Psi_{q}\right) \varphi_{i} \mathrm{~d} \Omega .
$$

Similarly, $\mathbf{A}^{q}$ is the vector of unknowns with elements

$$
\left\lfloor\mathbf{A}^{q}\right\rfloor_{i}=A_{i q} \text {. }
$$

Finally, the elements of the stiffness matrix are

$$
\left[\mathbf{S}^{q}\right]_{i, j}=\mathrm{E}\left(\Psi_{q} \Psi_{q}\right) \int_{\Omega}\left(v \nabla \varphi_{i}\right) \cdot\left(\nabla \varphi_{j}\right) \mathrm{d} \Omega .
$$

It can be seen that each order of the polynomial chaos expansion of $A$ forms its own system of $N$ equations, with no coupling between the systems of different order. Due to this property, the solution to the whole problem can be obtained by solving $P+1$ linear systems of $N$ equations, rather than one system of $(P+1) \times N$ equations. Furthermore, it can be seen that the matrix $\mathbf{S}^{q}$ is simply the stiffness matrix of the deterministic finite element method [14], multiplied with the expectation term. These factors considerably simplify the actual solution process, and decrease the necessary computation time.

\section{D. ssFEM Formulation in a Time-Harmonic Case}

If the conductor is connected to an AC voltage source, eddy currents induced in the conductor will change the governing equations slightly. In a time-harmonic deterministic case with sinusoidally varying variables, the problem can be expressed as

$$
\begin{aligned}
-\nabla \cdot(v(\mathbf{x}) \nabla A(\mathbf{x})) & +j \omega \sigma(\mathbf{x}) A(\mathbf{x}) \\
& =\frac{1}{l} \sigma(\mathbf{x}) U(\mathbf{x}),
\end{aligned}
$$

where $\omega$ is the supply frequency, $\sigma$ is the conductivity, $U$ is the supply voltage and $l$ is the effective length of the problem domain in the z-direction. [15] The second term is the induced eddy current density due to the time-varying vector potential. The right-hand side term is the non-eddy AC-current density due to the source voltage.

In a stochastic case, also the conductivity and the voltage will be random variables, due to the unknown conductor location. By assuming that the conductivity is constant over the conductor area and zero elsewhere, and that a constant voltage is applied over the ends of the conductor, the following two substitutions can be made in (23)

$$
\begin{array}{r}
\sigma(\mathbf{x}, \theta) \hat{=} \sigma \mathrm{D}_{\theta}(x, X(\theta)) \\
\sigma(\mathbf{x}, \theta) U(\mathbf{x}, \theta) \hat{=} \sigma U \mathrm{D}_{\theta}(x, X(\theta)) .
\end{array}
$$

In other words, the conductivity and the source voltage can be expressed with the same stochastic indicator function defined earlier. By again using

$$
g=\Psi^{q} \varphi_{n}
$$

as the test function, the following block matrix equation can be obtained

$$
\left[\begin{array}{cccc}
\mathbf{S}^{1}+\mathbf{S}_{\mathrm{e}}^{1,1} & \mathbf{S}_{\mathrm{e}}^{1,2} & \ldots & \mathbf{S}_{\mathrm{e}}^{1, P+1} \\
\mathbf{S}_{\mathrm{e}}^{2,1} & \mathbf{S}^{2}+\mathbf{S}_{\mathrm{e}}^{2,2} & \ldots & \mathbf{S}_{\mathrm{e}}^{2, P+1} \\
\vdots & \vdots & \ddots & \vdots \\
\mathbf{S}_{\mathrm{e}}^{P+1,1} & \mathbf{S}_{\mathrm{e}}^{P+1,2} & \ldots & \mathbf{S}^{P+1}+\mathbf{S}_{\mathrm{e}}^{P+1, P+1}
\end{array}\right]\left[\begin{array}{c}
\mathbf{A}^{1} \\
\mathbf{A}^{2} \\
\vdots \\
\mathbf{A}^{P+1}
\end{array}\right]
$$

$$
=\left[\begin{array}{c}
\mathbf{F}^{1} \\
\mathbf{F}^{2} \\
\vdots \\
\mathbf{F}^{P+1}
\end{array}\right]
$$

The elements of the block matrices $\mathbf{S}^{q}$ are as defined before in (22). They do not contribute outside the block diagonal due to 
the orthogonality of the polynomial chaoses, which renders the expectation in (22) zero elsewhere.

The eddy current matrices $\mathbf{S}_{\mathrm{e}}$ are due to the eddy current term of (23), and have the elements

$$
\left[\mathbf{S}_{\mathrm{e}}^{q_{r}, q_{c}}\right]_{i, j}=j \omega \sigma \int_{\Omega} \mathrm{E}\left(D_{\theta} \Psi_{q_{r}} \Psi_{q_{c}}\right) \varphi_{i} \varphi_{j} \mathrm{~d} \Omega .
$$

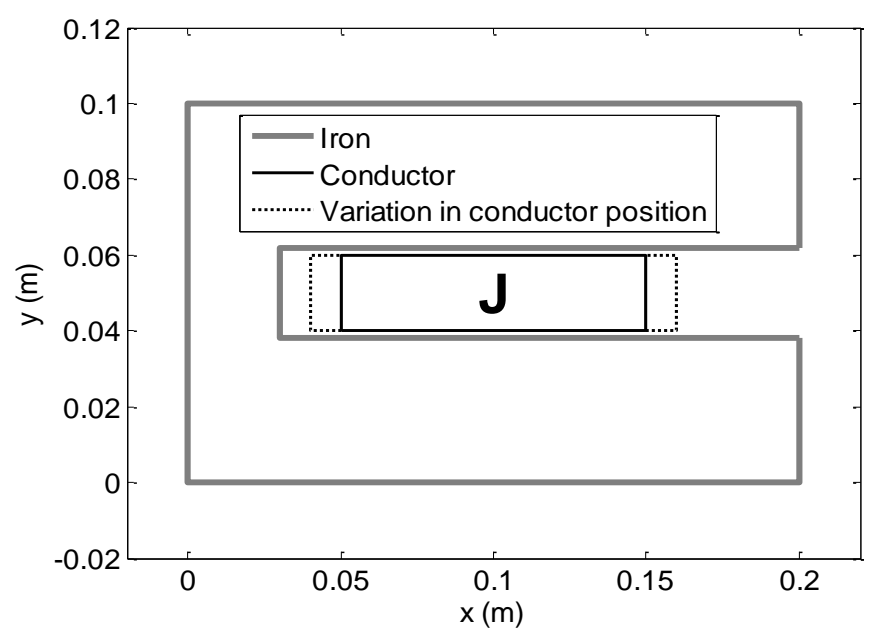

Fig. 1. The problem geometry illustrated.

In general, the eddy current matrices can be nonzero anywhere, depending on the indicator function and the polynomial chaos basis used.

The vectors of unknowns $\mathbf{A}^{q}$ are also as before, but the load vectors $\mathbf{F}^{q}$ are not. Instead, they have the elements

$$
\left[\mathbf{F}^{q}\right]_{i}=\frac{\sigma}{l} \int_{\Omega} \mathrm{E}\left(D_{\theta} \Psi_{q}\right) \varphi_{i} \mathrm{~d} \Omega .
$$

This time, the different-order coefficients $\mathbf{A}^{q}$ are generally coupled to each other by the eddy current matrices. Therefore, the problem cannot be split into smaller subproblems anymore. Instead, one large problem with $(P+1) \times N$ variables has to be solved. This can present a considerable increase in computation time compared to the static case.

\section{E. Interpreting the Results}

Once the matrix equations have been solved, statistical behaviour of the vector potential can be easily calculated. For instance, the mean value of $\mathrm{A}$ at each node $i$ can be calculated with

$$
\mathrm{E}\left(A_{i}\right)=\mathrm{E}\left(\sum_{j=0}^{P} A_{i j} \Psi_{j}\right)=A_{i 0} \mathrm{E}\left(\Psi_{0}\right) .
$$

Higher order terms have been dropped from the expression because for all $j \geq 0$ it holds that

$$
\mathrm{E}\left(\Psi_{j}\right)=c \mathrm{E}\left(\Psi_{j} \Psi_{0}\right)=0
$$

where $c$ is some scalar, due to the orthogonality of the chaoses. Similarly, the variance of the vector potential can be obtained from

$$
\begin{aligned}
& \operatorname{Var}\left(A_{i}\right)=\mathrm{E}\left(A_{i}^{2}\right)-\mathrm{E}\left(A_{i}\right)^{2} \\
& =\mathrm{E}\left(\left(\sum_{j=0}^{P} A_{i j} \Psi_{j}\right)^{2}\right)-A_{i 0}^{2} \mathrm{E}\left(\Psi_{0}\right)^{2} \\
& =\sum_{j=1}^{P} A_{i j}^{2} \mathrm{E}\left(\Psi_{j}^{2}\right) .
\end{aligned}
$$

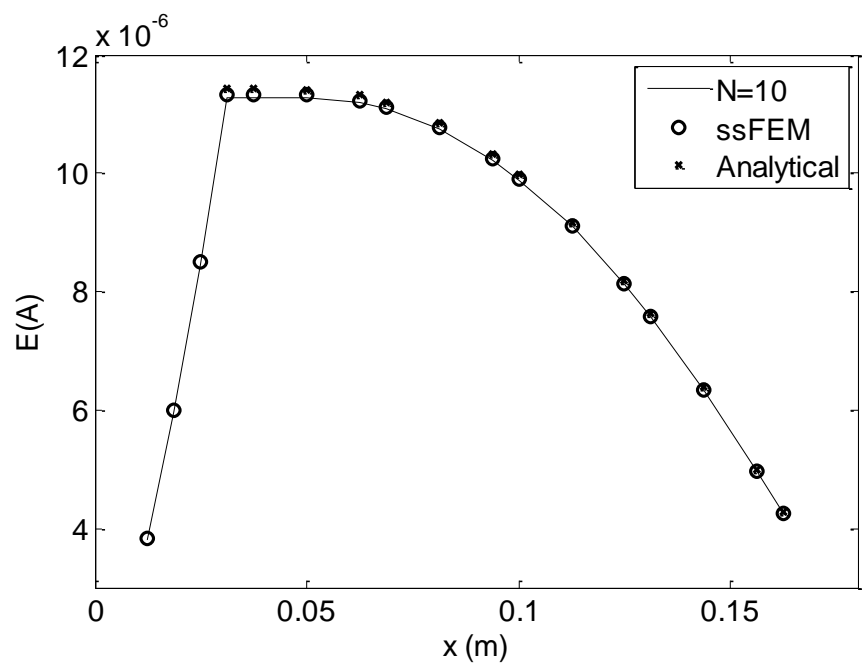

Fig. 2. The mean value of the vector potential at (x, 0.05) obtained with the Monte-Carlo method, the proposed ssFEM method and the analytical approximation.

In the final form, the zero-order term has been dropped since

$$
\mathrm{E}\left(\Psi_{0}^{2}\right)=\mathrm{E}\left(\Psi_{0}\right)^{2}
$$

Similarly, most of the expectation terms have vanished due to the orthogonality of the polynomial chaoses.

Similar formulae can be written for higher moments as well, should it be desired. Indeed, one of the main benefits of the spectral stochastic FEM is the easy calculation of variances and higher moments, compared to for instance the Monte Carlo method [7].

\section{Simulation Results}

A simplified problem geometry was chosen to be simulated both with the proposed stochastic finite element formulation and with the Monte Carlo method. Both the cases with and without eddy currents were tested. The results were then compared to each other, as well as analytically obtained values in the case with no eddy currents.

\section{A. Conductor in a Slot with Constant Current Density}

A very simple geometry, shown in Fig 1 was selected to test the proposed method without eddy currents. The problem domain consisted of a $0.1 \mathrm{~m} \times 0.05 \mathrm{~m}$ conductor in a slot. The x-position of the conductor's center point was chosen to be random, distributed uniformly between $[0.09,1.01]$. The iron parts were assumed to be linear, with a relative permeability of 1000. The domain was then discretized into 2048 first-order elements with 1089 nodes. First-order shape functions were used. The polynomial chaos expansion of the vector potential 
was then solved with the proposed method. Hermite polynomials were used as the bases of the polynomial chaos.

Two methods were used for verification. Firstly, numerous Monte Carlo simulations were performed with the deterministic finite-element method with the conductor in a randomly selected position in each case. Secondly, an approximate expression for the vector potential in the slot was derived. With this method, it was assumed that the iron parts

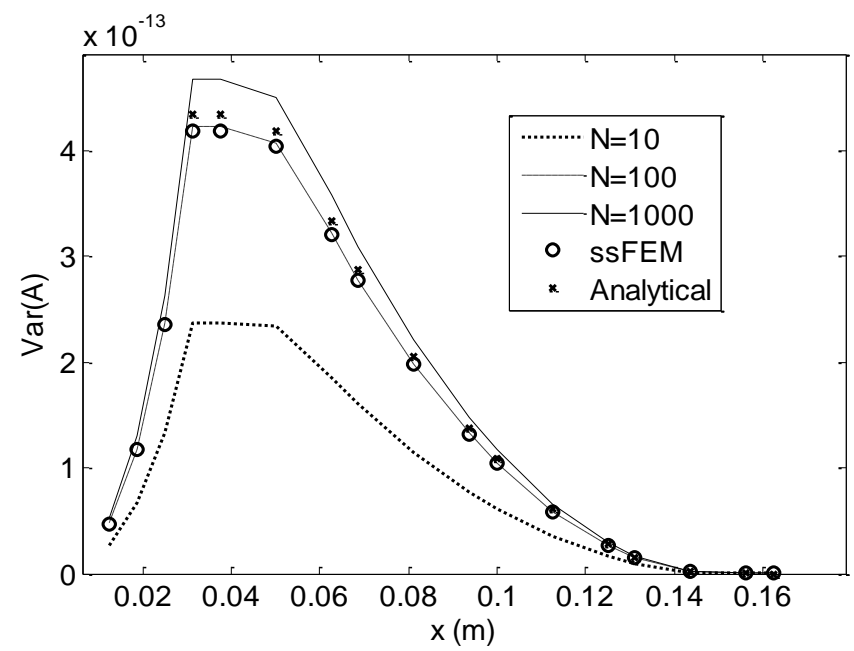

Fig. 3. Variances at $(\mathrm{x}, 0.05)$ obtained with different Monte-Carlo simulations, ssFEM and analytically.

had an infinite permeability and that the flux travelled purely in the y-direction in the slot.

Fig 2 shows the mean values of the vector potential in the centre of the slot, along the line $y=0.05 \mathrm{~m}$. The solid line shows the results obtained with 10 Monte Carlo simulations. The circles show the results from the proposed ssFEM method, while the analytical solutions are marked with the crosses. A tenth-order polynomial expansion was used with ssFEM.

It can be seen that all three methods produce very similar results. The ability of the Monte Carlo method to produce such accurate results with only ten simulations is slightly surprising. Nevertheless, with the proposed stochastic finite element method it would have been sufficient to limit the approximation to mean values only, i.e. set $P$ to 0 , and the same result would have been obtained. Still, in this case the Monte Carlo method appears to be a very tempting competitor to the more complex ssFEM.

More differences come about with variance calculation. Fig 3 shows the variances of the vector potential along the same line, obtained with the three described methods. The three different lines show the results obtained with 10, 100 and 1000 Monte Carlo runs, respectively. Circles again mark the ssFEM results obtained with $P=10$, while analytical results are plotted with the crosses once again.

This time, much clearer differences between the results can be seen. Also here, the ssFEM and analytical results are reasonably close to each other. However, the variance obtained with 10 Monte Carlo runs is nowhere near the other two methods. With 100 runs, a good approximation is obtained, although the again-diverging result from 1000 calculations suggests this was due to chance only. Indeed, it was observed that the Monte Carlo method converged near the ssFEM and analytical results only after 10000 simulations or more.

This slow convergence of the variance clearly signifies the benefits of the spectral stochastic FEM against the Monte Carlo method. While relatively good approximations of the mean solution might easily be obtained with the Monte Carlo

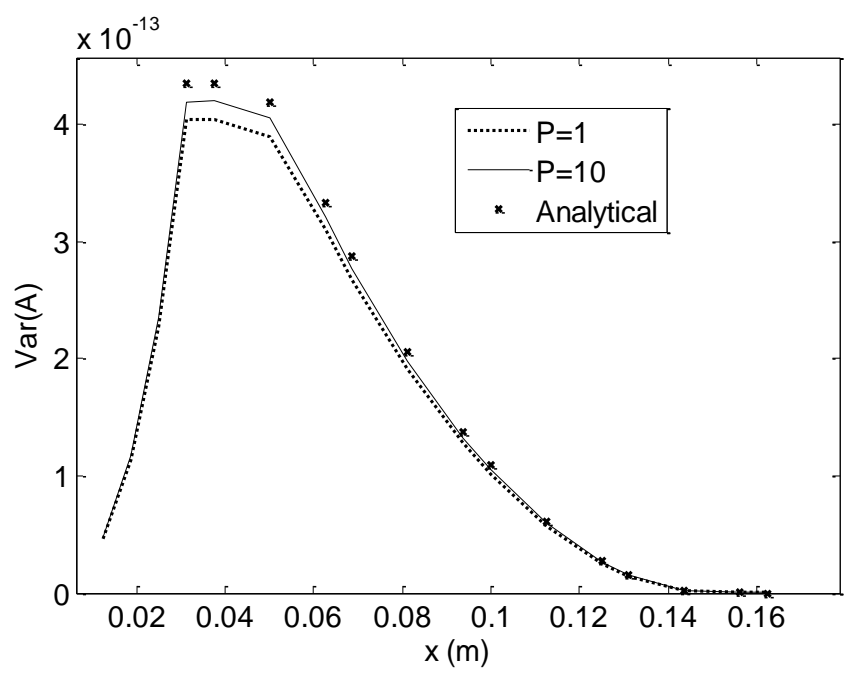

Fig. 4. Variance convergence with ssFEM.

method, this is often not the case for variances, let alone any higher moments. Instead, a lot of repeated simulations are usually necessary for a reliable result. By contrast, with the ssFEM it is sufficient to solve one larger problem once.

It may also be of interest to see the convergence of ssFEMcalculated variances as the order of approximation $\mathrm{P}$ is increased. For this purpose, Fig 4 shows three approximated variances along the same $y=0.05$ line as previously. Analytical results are marked with the crosses, while the two lines show the ssFEM results. The dotted line shows the variance obtained with a first-order polynomial chaos expansion, while the solid line shows the result with $P=10$. It can be seen that in this case, even a first order expansion yields relatively good results. Moreover, a large increase in the order of approximation does not yield any significant additional improvement in accuracy.

\section{B. Conductor in a Slot with Eddy Currents}

The same problem geometry was simulated again, only this time eddy currents were allowed to flow in the conductor. Furthermore, the conductor was allowed to be anywhere between the slot bottom and the slot opening.

Uniform conductor position distribution in the $\mathrm{x}$-direction was again used. A supply voltage of $10 \mathrm{~V}$ per one meter of conductor length was used, and the supply frequency was set to $50 \mathrm{~Hz}$. The conductor was chosen to be made of copper. These parameters were chosen mainly for illustrative purposes, e.g. to obtain a reasonable balance between conductor inductance and resistance. Legendre polynomials were used with the chaos expansion. 


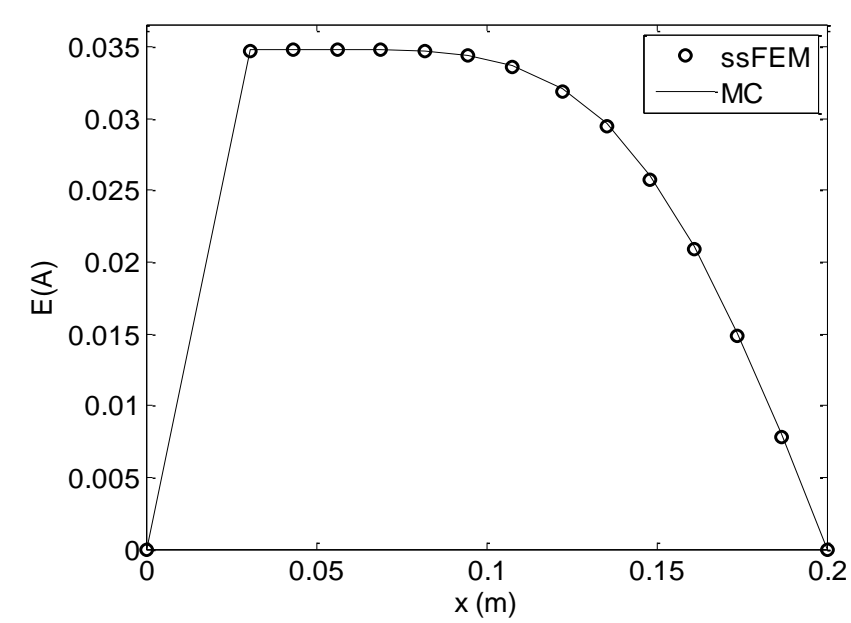

Fig. 5. Mean vector potential (eddy current case) at (x, 0.05) obtained with the Monte Carlo method and with ssFEM.

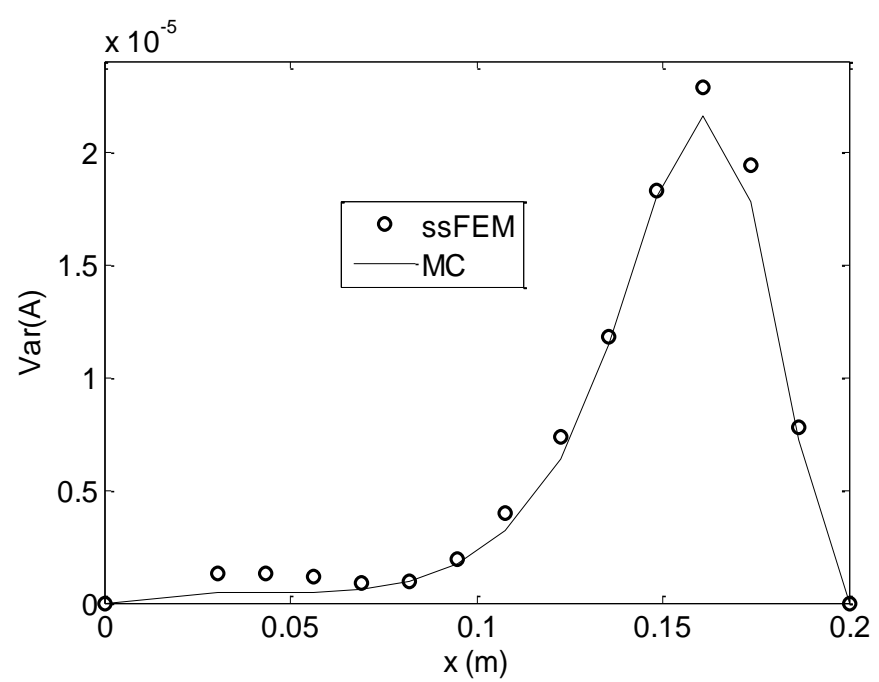

Fig. 6. Variance of the vector potential at $(\mathrm{x}, 0.05)$ obtained with the Monte Carlo method and ssFEM.

With this problem, only the ssFEM and the Monte Carlo method were chosen to be compared, mainly due to the difficulty of obtaining analytical solutions to eddy current problems. Fig 5 shows the expected absolute values of the vector potential along the same $(x, 0.05)$ line as before. Monte Carlo results from 1000 simulations are shown with the solid line, while the circles show the ssFEM results with $P=5$. Again, a very good agreement can be observed between the different methods.

Although this is not visible in the figure, additional simulations revealed that very similar results could also be obtained much faster, i.e. with a smaller number of Monte Carlo runs, or with a smaller order of approximation $\mathrm{P}$ in the ssFEM. In other words, accurate estimates for the mean values were easy to obtain also in this case.

Fig 6 shows the variances of the vector potential along the same line. Again, Monte Carlo results are marked with the solid line, while the circles denote results by ssFEM. This time, more differences can be observed, both near the peak and also around $x=0.05$. In general, the Monte Carlo method seems to predict lower variances than the stochastic finite element method does. However, it cannot be deduced from the results whether this due to inaccuracies in the ssFEM, limited convergence of the Monte Carlo method, or both. Nevertheless, a reasonable agreement has indeed been obtained in this case as well.

Besides the values of the vector potential, also the induced eddy currents may be of interest. Therefore, effective values of the total induced current were calculated with the expression

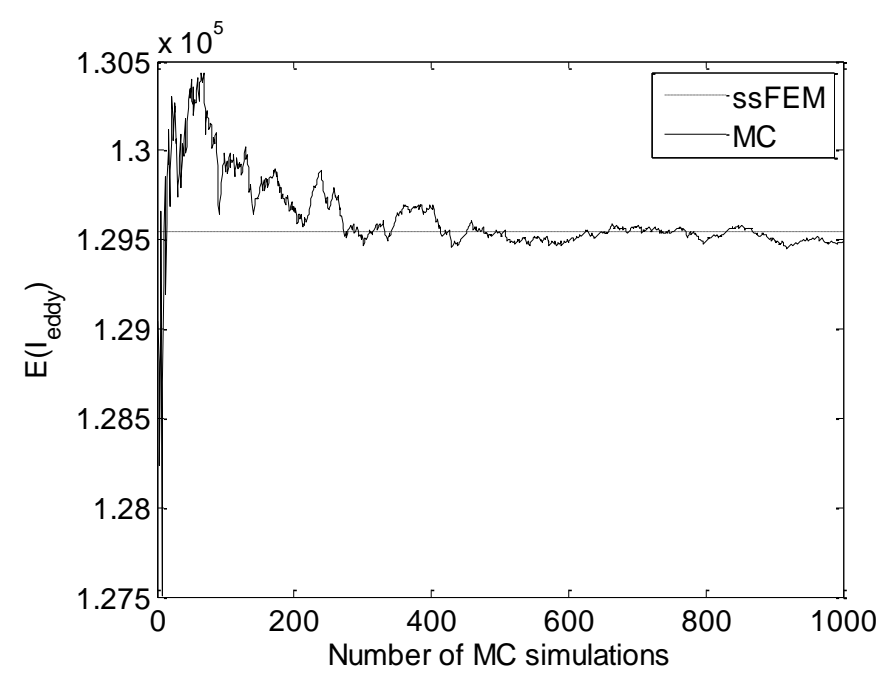

Fig. 7. Mean values of the total induced eddy currents calculated with the Monte Carlo method and with ssFEM.

$$
\left|\int_{\Omega} j \omega \sigma(\mathbf{x}) A(\mathbf{x}) \mathrm{d} \Omega\right|,
$$

and the results were then plotted in Fig 7. The solid line shows the convergence of the induced current as a function of the number of Monte Carlo simulations performed. The dotted horizontal line shows the result from the single ssFEM solution. It can be seen that more than 400 Monte Carlo simulations are necessary to obtain a reasonable agreement between the two methods. Even after 1000 iterations, the Monte Carlo method has yet to fully converge, i.e. there are still some visible fluctuations.

Indeed, in this case the stochastic finite element method appears to be much faster even for mean value calculation, instead of only variances like was the case in the previous problem. This change in behaviour is probably caused by the more sensitive coupling between the inducing vector potential and the induced current density.

Because of this interaction, any inaccuracy in either of these quantities will cause inaccuracies in the other. In the earlier simulation, the current density was fixed and this interaction therefore missing, which most probably explains the large difference in convergence speeds.

Finally, Fig 8 shows the convergence of the variance of the induced current as a function of the number of simulations. Again, the variance from ssFEM calculations is shown with the dotted line. 


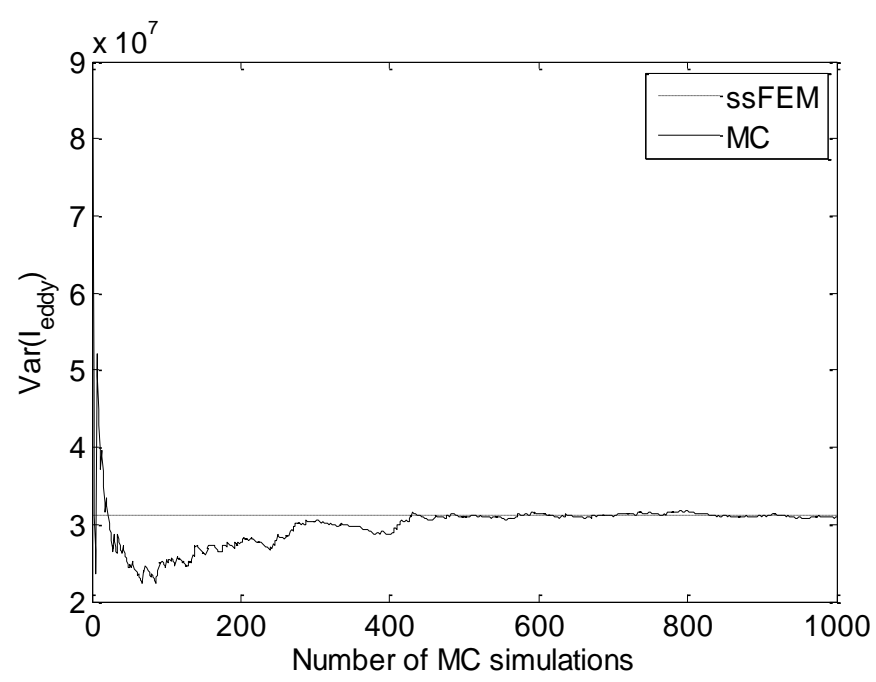

Fig. 8. Variances of the induced eddy currents calculated with the Monte Carlo method and with ssFEM.

Surprisingly, this time the variance seems to converge faster than the mean value of Fig 7; after 500 simulations there are few fluctuations. A nice agreement is found between the two methods. However, based on some additional simulations, the fast variance convergence of the Monte Carlo method seems to have been due to chance only, and specific to this particular problem. With even slightly different problem parameters, the variance of the current converged slower than the mean value in a majority of cases.

\section{Observed Limitations}

While solving the example problem with a constant current density was very straightforward, some difficulties were encountered in the eddy current case. Apparently, the eddy current problem is to be relatively sensitive to the mesh used. Indeed, large differences were observed between the Monte Carlo method and the proposed ssFEM with a coarse mesh. This phenomenon is due to a coarse mesh failing to pick up more subtle variations in the conductor position. Further study is required to determine which method actually gives more realistic result in these problematic cases.

By using a mesh with a relatively fine division in the $x$-direction over the slot area, the differences could be minimized. In the example problem with eddy currents, the slot area was divided into 80 slices and meshed accordingly to obtain the best possible results.

\section{On convergence speeds}

It must be noted the number of Monte Carlo simulations necessary to match the accuracy of the ssFEM method may be slightly misleading, since there are $(P+1)$ times more unknowns in the spectral method. Nevertheless, in all the example problems good solutions were obtained with $P=3 \ldots$. 5. Since a non-optimized solution of a linear system is a $\mathrm{O}\left(n^{3}\right)$ operation, this corresponds to a $64 . .216$-fold increase in computation times. Obviously, this will still be significantly faster than performing $\geq 1000$ Monte Carlo runs, as was necessary in most cases. Furthermore, the stiffness matrices of the stochastic systems will be relatively sparse, so faster solutions could most probably be obtained by iterative means.

\section{CONCLUSIONS}

Stochastic electromagnetic problems have usually been solved either with the Monte Carlo method or some stochastic finite element method. Of these alternatives, the Monte Carlo method usually requires a very large amount of computation time. The spectral stochastic finite element method is usually a faster alternative, but has until now been mainly used to solve problems with uncertainties in the material characteristics rather than the problem geometry.

This paper proposes an indicator-function based method to solve two-dimensional electromagnetic problems with conductors on random locations. The conductor position is expressed with a stochastic indicator function that takes into account the uncertainty. The rest of the problem is then solved as with typical spectral stochastic methods. Equations of a single-conductor case both with and without eddy currents are presented in this paper. Generalizing the method for several conductors should be fairly trivial, provided that the conductors are in separate slots or otherwise cannot overlap. An overlapping case is a subject of the author's further study. The proposed method could possibly be used also for other than electromagnetic problems, though this has not been demonstrated yet.

The proposed method was used to solve a simple singleconductor example case, both with and without eddy currents. Mean values and variances of the vector potential and the total induced eddy currents were calculated. The results were verified with the well-established Monte Carlo method, and also with a simple analytical method in the case with no eddy currents. A very good agreement was found in most cases, although there were some unexplained differences in the variance of the vector potential in the eddy current problem.

Significantly faster solutions were observed with the proposed method compared to the Monte Carlo method. In many cases, the Monte Carlo method required several hundred simulations to converge properly. This seemed to be the case especially when calculating variances of the problem quantities. It is reasonable to assume that these differences in computation times would be compounded when calculating third or higher moments.

The proposed method appears to be relatively sensitive to the meshing of the problem region, at least if the effects of eddy currents are included. The cause of this sensitivity remains unknown. Solving this limitation would require some further study. Nevertheless, it appears that good solutions can still be obtained without excessively fine meshes, provided that the mesh has been appropriately refined in the random regions of the problem domain. Since mesh-generation is in any case a highly problem-specific problem, this requirement cannot be considered a serious drawback.

Generally, this paper stands as a demonstration that the spectral stochastic finite element method can easily be modified to solve problems with geometric uncertainties as well as material ones. In electrical engineering, one obvious 
problem of such nature is the demonstrated conductor position case. However, the method could probably also be extended to other fields as well, such as modelling random structural deformations.

\section{V.ACKNOWLEDGMENTS}

Financial support from Emil Aaltonen Foundation, Finland, is gratefully accepted.

\section{REFERENCES}

[1] G. Crevecoeur, P. Sergeant, L. Dupré and L. Vandenbossche, "Analysis of the local material degradation near cutting edges of electrical steel sheets," IEEE Transactions on Magnetics, vol. 44, no. 11, pp. 3173-3176, 2010. http://dx.doi.org/10.1109/TMAG.2008.2001605

[2] J. Lähteenmäki, Design and voltage supply of high-speed induction machines, Acta Polytechnica Scandinavica, Electrical Engineering series no. 108, Espoo 2002.

[3] P.B. Reddy and T.M. Jahns, "Analysis of bundle losses in high speed machines," 2010 International Power Electronics Conference (IPEC), pp. 2181-2188, 2010. http://dx.doi.org/10.1109/IPEC.2010.5542354

[4] A.S. Thomas, Z.Q. Zhu and G.W. Jewell, "Proximity loss study in high speed flux-switching permanent magnet machine," IEEE Transactions on Magnetics, vol. 45, issue 10, pp. 4748-4751, 2009. http://dx.doi.org/10.1109/TMAG.2009.2021666

[5] P.H. Mellor, R. Wrobel and N. McNeil, "Investigation of Proximity Losses in a High Speed Brushless Permanent Magnet Motor", Conference Record of the 2006 IEEE Industry Applications Conference, 2006. 41st IAS Annual Meeting, vol. 3, 1514-1518, 2006.

[6] J. Fang, X. Liu, B. Han and K. Wang, "Design aspects of winding for low-voltage high-speed permanent magnet motor," IEEE Transactions on Energy Conversion (in review), 2014.

[7] R.G. Ghanem and P.D. Spanos, Stochastic Finite Elements - A Spectral Approach. New York: Springer Verlag, 1991. http://dx.doi.org/10.1007/978-1-4612-3094-6

[8] R. Gaignaire, S. Clenet, B. Sudret, and O. Moreau, "3-D spectral stochastic finite element method in electromagnetism," IEEE Transactions on Magnetics, vol. 43, no. 4, pp. 1209-1212, 2007. http://dx.doi.org/10.1109/TMAG.2007.892300
[9] K. Beddek, Y. Le Menach, S. Clenet, and O. Moreau, "3-D stochastic spectral finite-element method in static electromagnetism using vector potential formulation," IEEE Transactions on Magnetics, vol. 47, no. 5, pp. 1250-1253, 2011. http://dx.doi.org/10.1109/TMAG.2010.2076274

[10] R. Gaignaire, S. Clenet, O.Moreau and B.Sudret, "Current calculation in electrokinetics using a spectral stochastic finite element method," IEEE Transactions on Magnetic, vol. 44, issue 6, pp. 754-757, 2008. http://dx.doi.org/10.1109/TMAG.2008.915801

[11] D.H. Mac, S. Clénet, J.C. Mipo and O. Moreau, "Solution of static field problems with random domains," IEEE Transactions on Magnetics, vol. 2, no. 8, pp. 3385-3388, 2010.

[12] D.H. Mac, S. Clénet and J.C. Mipo, "Calculation of field distribution in electromagnetic problems with random domains," IET 8th International Conference on Computation in Electromagnetics (CEM), pp. 1-2, 2011.

[13] B.J. Debusschere, H.N. Najm , P. Pebay , O.M. Knio , R.G. Ghanem and O. Le Maitre, "Numerical challenges in the use of polynomial chaos representations for stochastic processes," SIAM Journal of Scientific Computing, vol. 26, no. 2, pp. 698-719, 2004. http://dx.doi.org/10.1137/S1064827503427741

[14] A. Arkkio, Analysis of induction motors based on the numerical solution of the magnetic field and circuit equations, Acta Polytechnica Scandinavica, Electrical Engineering series no. 59, Espoo 1987.

[15] M.J. Islam, J. Pippuri, J. Perho and A. Arkkio, "Time-harmonic finiteelement analysis of eddy currents in the form-wound stator winding of a cage induction motor," IET Electric Power Applications, vol. 1, no. 5, pp. 849-846, 2007.

Antti Lehikoinen was born in December 1988. He received his bachelor's degree in science (technology) in 2012 from the Aalto University School of Electrical Engineering, and the M. Sc. degree a year later from the same institute, with a major in electromechanics and minor in drives.

$\mathrm{He}$ is currently making his doctoral thesis in the School of Electrical Engineering, Espoo, Finland. He is mainly focusing on stochastic modelling and prediction of additional losses due to circulating currents in randomwound machines.

Address: Otakaari 5, 02120 Espoo, Finland, Aalto University School of Electrical Engineering

E-mail: antti.lehikoinen@aalto.fi 